\title{
EXPRESSION OF Ki-67 AND CD105 MARKERS IN ORAL SQUAMOUS CELL CARCINOMA
}

\author{
Raghda M. Abdelbary ${ }^{1} B D S$, Mohamed S. Elzarka² PhD, Hamed A. Fouad ${ }^{2} P h D$, Mohammed \\ M. Fata ${ }^{3} P h D$.
}

\begin{abstract}
INTRODUCTION: Oral squamous cell carcinoma (OSCC) is the commonest malignancy in the oral cavity. It is influenced by genetic modifications; as well as numerous environmental factors, such as smoking, tobacco chewing, alcohol consumption, infection by oncogenic viruses, and low intake of fruits and vegetables. CD105 has been introduced as a marker for angiogenesis which stains vessels that are in the proliferating stage. Ki-67is a nuclear protein that is recognized as a nuclear antigen present in proliferating cells, but absent in resting cells. OBJECTIVES: To evaluate and correlate the expression of Ki-67 and CD105 in different histopathological grades of OSCC.

MATERIALS AND METHODS: Immunohistochemical (IHC) study using the Ki-67 and CD105 antibody was done on 20 surgical specimens and 5 normal mucosal tissues taken from OSCC patients and healthy individuals respectively.

RESULTS: Remarkable improvement in the histology and the ultrastructure of the alveolar bone of rats in Group III was observed. Moreover, hematological values revealed significant decrease in the inflammatory condition of rats with induced RA after fish oil treatment.

CONCLUSIONS: Ki-67 and CD105 are expressed in OSCC biopsies. Additionally, the fraction of Ki-67positive tumor cells (the Ki-67 labeling index) is often correlated with the clinical course of OSCC. CD105 microvessel density can be a useful factor for predicting the course
\end{abstract} of oral squamous cell carcinoma

KEYWORDS: Oral Squamous Cell Carcinoma, Ki-67, CD105, Imunohistochemistry, Angiogenesis.

1. B.D.S 2009

2. Professor of Oral Pathology, Faculty of Dentistry, Alexandria University

3. Professor in Cranio Maxillofacial and Plastic Surgery, faculty of dentistry, Alexandria University.

Corresponding author:

E-mail:r.m.abdelbary@gmail.com

\section{INTRODUCTION}

Oral cancer is a global health problem. Around 300,000 patients are annually estimated to have this disease worldwide. Our main interest falls in head and neck squamous cell carcinoma (HNSCC) in general and oral squamous cell carcinoma (OSCC) in specific because of its alarming incidence and mortality (1).

Oral squamous cell carcinoma is a multifactorial disease. It is influenced by genetic modifications; as well as numerous environmental factors, such as smoking, tobacco chewing, alcohol consumption, infection by oncogenic viruses, and low intake of fruits and vegetables (2).

Angiogenesis is fundamental in tumor growth, progression and metastasis (3). The growth of a solid tumor is closely connected to the development of an intrinsic vascular network. In addition to primary tumor growth, metastatic tumor growth depends upon neovascularization in at least two steps: First, malignant cells must exit from a primary tumor into the blood circulation after the tumor becomes neovascularized. Second, after arrival at distant organs, metastatic cells must again induce angiogenesis for a tumor to expand to a detectable size (4).

Cell proliferation is a biological process vitally important to all living organisms due to its role in the growth and maintenance of tissue homeostasis. The assessment of cell proliferation activity in tumors has become a common tool used by histo-pathologists in order to provide useful information for diagnosis, clinical behavior, and therapy (5).

CD105 (endoglin) is predominantly transmembrane glycoprotein expressed in angiogenic endothelial cells (EC) and up-regulated by hypoxia (6). It is expressed on the cell surface. It is an accessory protein of the transforming growth factor- $\beta$ (TGF-beta) receptor system and composed of two disulfide-linked subunits of $95 \mathrm{kDa}$ each forming a 180-kDa homodimeric mature protein(6).

The Ki-67 is a nuclear non-histone protein, strictly associated with cell proliferation. Playing roles in cell cycle regulation, synthesis of ribosomes and also being related to survival, malignancy and prognosis of various neoplasms, including OSCC. With unknown chemical structure and molecular weight ranging from 345 to $395 \mathrm{kDa}$. It is encoded by a gene located on chromosome 10q25, containing phosphorylation sites for a variety of kinases (7, 8).

The aim of the present study was to study the expression of Ki-67 and CD 105 in oral squamous cell carcinomas and to correlate the expression of Ki-67 and CD 105 in different grades of these tumors.

\section{MATERIALS AND METHODS}

The study was performed in the Faculty of Dentistry, Alexandria University after gaining the approval of the Research Ethics Committee. Twenty OSCC patients collected from the Cranio-Maxillofacial and Plastic Surgery Department were included. As well as, five age and gender matched non-cancer patients who were undergoing minor oral procedures selected from the Oral and Maxillofacial Surgery Department serving as negative control. All patients signed informed consents for the agreement to participate in the study. The patients and controls who presented with signs of active infection, autoimmune diseases, or having a history of radiotherapy, chemotherapy or other cancers were excluded from the study. 
Biopsies were taken from the tumor tissues in cancer patients as well as from normal mucosal tissues taken from normal mucosal tissues taken from healthy individuals. The specimens were fixed in $10 \%$ neutral buffered formalin, processed and embedded in paraffin wax using the conventional procedures. Serial sections of 3-4 $\mu \mathrm{m}$ thickness were mounted on glass slides and stained using Hematoxylin and Eosin (H\&E). Immunohistochemical (IHC) staining using the Ki-67 and CD 105 antibody (Thermo Fisher Scientific, USA) was also performed using the Labeled Strept- Avidin Biotin complex method (LSAB). Then, the sections were examined by the image analyzer computer system using the software Leica Qwin 500.

\section{Statistical analysis}

Analysis of data was performed using SPSS 17 (Statistical Package for Scientific Studies) for Windows.

Description of quantitative variables was in the form of mean and standard deviation (SD).

- The mean values of area percent and optical density of Ki67 and CD105 expression in different histological grades were compared by using analysis of variance (ANOVA) test.

- Student's (t) test was used for comparison between expression of Ki-67 and CD105 in OSCC cases.

- Results were expressed in the form p-values that were differentiated into:

- Non-significant when p-value $>0.05$.

- Significant when p-value $\leq 0.05$.

- Highly significant when p-value $\leq 0.01$.

\section{RESULTS}

\section{Clinical Results}

The demographic data of the patients included in the study were shown in (Table 1). The age of the patients included in this study ranged between 51 and 70 years. years. Thirteen patients (65\%) were males and seven patients (35\%) were females.

Table (1): The clinical pathologic parameters of studied cases of OSCC.

\begin{tabular}{|c|c|c|}
\hline Clinical Demographic & No & $\%$ \\
\hline $\begin{array}{l}\text { Gender } \\
\text { Male } \\
\text { Female }\end{array}$ & $\begin{array}{c}13 \\
7 \\
\end{array}$ & $\begin{array}{l}65.0 \\
35.0\end{array}$ \\
\hline $\begin{array}{l}\text { Age } \\
\geq 60 \\
<60\end{array}$ & $\begin{array}{c}11 \\
9\end{array}$ & $\begin{array}{l}55.0 \\
45.0\end{array}$ \\
\hline Min. - Max. & \multicolumn{2}{|c|}{$51.0-70.0$} \\
\hline Mean \pm SD & \multicolumn{2}{|c|}{$60.75 \pm 6.62$} \\
\hline Median & \multicolumn{2}{|c|}{60} \\
\hline $\begin{array}{c}\text { Site } \\
\text { Tongue } \\
\text { Alveolar mucosa } \\
\text { Buccal mucosa } \\
\text { Palatal mucosa } \\
\text { Retromolar area } \\
\text { Lip } \\
\end{array}$ & $\begin{array}{l}6 \\
5 \\
4 \\
2 \\
2 \\
1 \\
\end{array}$ & $\begin{array}{c}30.0 \\
25.0 \\
20.0 \\
10.0 \\
10.0 \\
5.0 \\
\end{array}$ \\
\hline $\begin{array}{c}\text { Lymph Node Metastasis } \\
\text { Negative } \\
\text { Positive } \\
\end{array}$ & $\begin{array}{c}11 \\
9 \\
\end{array}$ & $\begin{array}{l}55.0 \\
45.0 \\
\end{array}$ \\
\hline $\begin{array}{c}\text { Clinical Appearance } \\
\text { Exophytic } \\
\text { Ulcerative }\end{array}$ & $\begin{array}{c}15 \\
5\end{array}$ & $\begin{array}{l}75.0 \\
25.0\end{array}$ \\
\hline
\end{tabular}

The most common site of occurrence of OSCC was the lateral side of the tongue (30\%), followed by the alveolar ridge (25\%), then buccal mucosa (20\%). Finally, The palatal mucosa and retromolar area each representing (10\%) and the least site was the lip representing (5\%)

\section{Histopathological Results}

The microscopical examination revealed that $25 \%$ of the cases were well differentiated, $65 \%$ were moderately differentiated and $10 \%$ were poorly differentiated.

\section{Immunohistochemical Results}

All cases of normal oral mucosa $(n=5)$ showed Positive immunoreactivity for Ki-67 and CD105 (Figure 1), while OSCC biopsies $(n=20)$ were immunostained. Both cytoplasmic and nuclear immunoreactions were observed in all different grades of OSCC (Figures 2, 3, 4, 5, and 6).

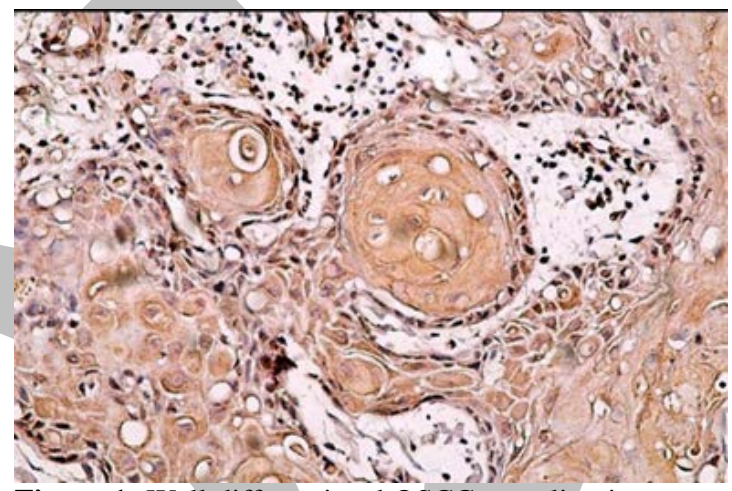

Figure 1: Well differentiated OSCC revealing intense reaction to ki67 in almost all of the malignant epithelial cells forming the epithelial and keratin pearls. (Immunostain of ki67 x200)

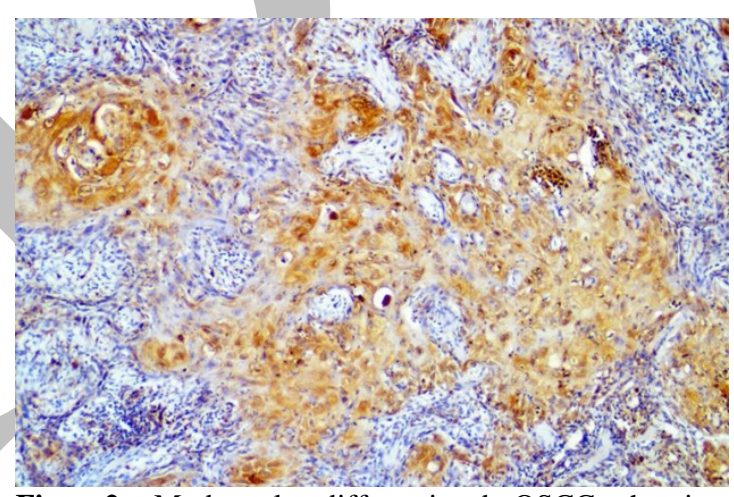

Figure2: Moderately differentiated OSCC showing evident positive reaction to ki67 in almost all malignant epithelial pearls and cell nests. Notice the apoptotic cell in the center exhibiting perinuclear cytoplasmic clearing (Immunostain of ki67 x100).

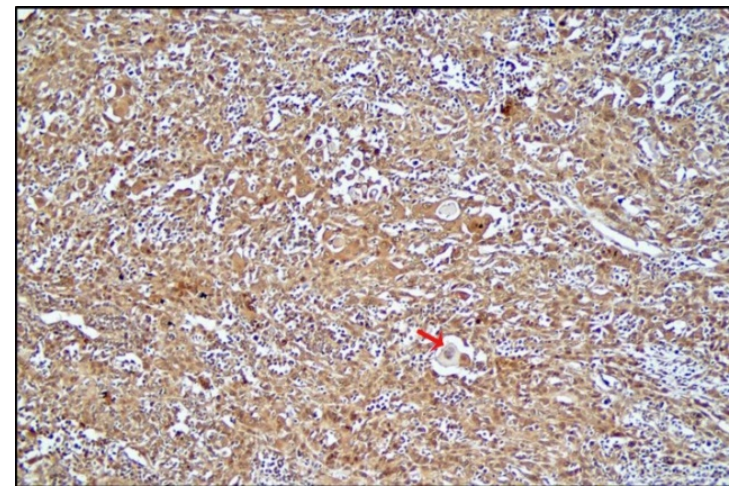

Figure 3: Poorly differentiated OSCC showing intense positive reaction of ki67 in the anaplastic cells forming the sheets of malignant epithelial cells. Notice the apoptotic cells clearance of the cytoplasm and chromatin condensation (arrow). (Immuno stain of ki67 x 100). 


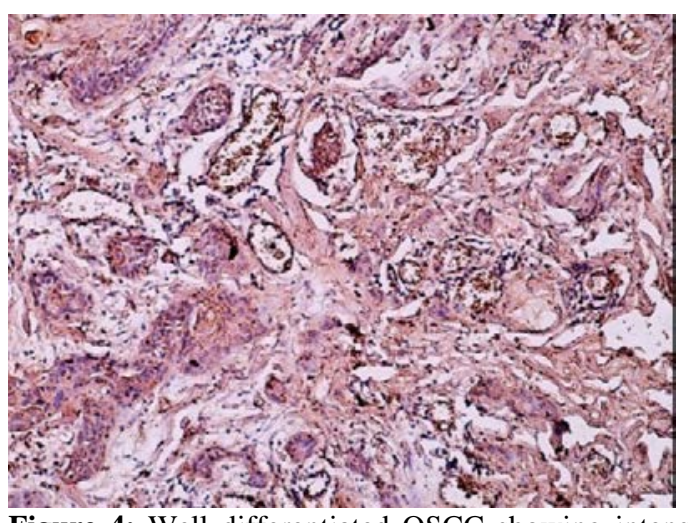

Figure 4: Well differentiated OSCC showing intense brownish immune reaction to CD105 of blood vessels and endothelial cells with some epithelial nests free from the reaction (immunostain of CD105 x100).

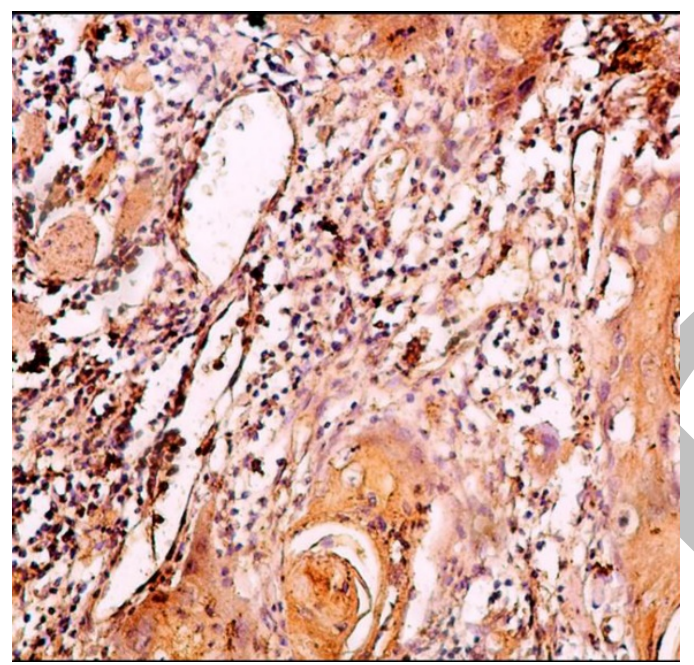

Figure 5: Moderately differentiated OSCC showing intense brownish immune reaction to CD105 of blood vessels and endothelial cells (immunostain of CD105x200).
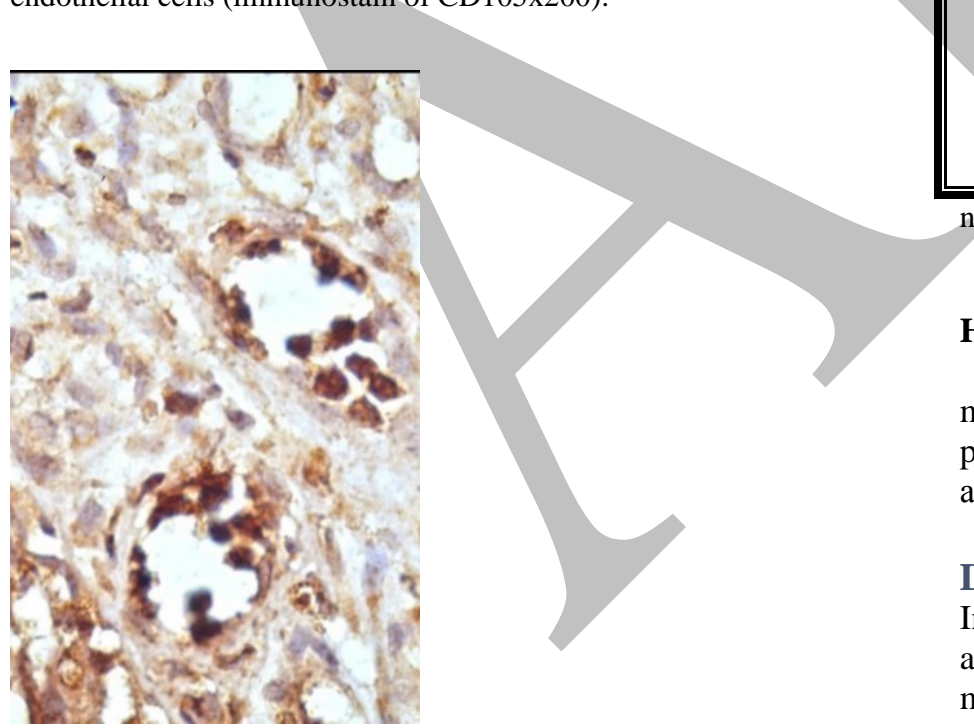

Figure 6: Moderately differentiated OSCC showing intense brownish immune reaction to CD105 at the stained blood vessels and endothelial cells (immunostain of CD105 x1000).

The IHC staining intensity was found to be directly proportional to the grading of OSCC, where the poorly differentiated type has the highest staining intensity, followed by the moderately and the well differentiated grades respectively.

Ki-67 expression in Correlation with the Histological Grading of OSCC cases
The difference in the mean Ki-67 area percent and mean optical density between the well, moderately and poorly differentiated groups revealed statistical significance at $(\mathrm{P} \leq 0.05)$ (Tables 2,3$)$.

Table (2): Comparison of area percent and optical density of Ki 67 in different histopathological grades.

\begin{tabular}{||l|l|l|l|l|l||}
\hline $\begin{array}{l}\text { Recept } \\
\text { or }\end{array}$ & $\begin{array}{l}\text { Point of } \\
\text { compar } \\
\text { ison }\end{array}$ & Well diff. & $\begin{array}{l}\text { Moderate } \\
\text { diff. }\end{array}$ & $\begin{array}{l}\text { Poorly } \\
\text { diff. }\end{array}$ & $\begin{array}{l}\text { Palue } \\
\text { (ANO } \\
\text { VA } \\
\text { test) }\end{array}$ \\
\hline & $\begin{array}{l}\text { Area } \\
\text { percent } \\
\text { Min. }-\end{array}$ & $11.34-$ & $26.48-$ & $37.86-$ & 0.001 \\
& & & & \\
& Max. & 31.26 & 35.96 & 49.02 & \\
& Mean \pm & $21.35 \pm 5$. & $31.32 \pm 4.2$ & $43.56 \pm 5$ & \\
Ki 67 & SD. & 16 & 8 & .96 & \\
& Optical & & & & \\
& Density & & & & \\
& Min. - & $42.68-$ & 45.57 & $57.56-$ & 0.017 \\
& Max. & 50.87 & 63.67 & 70.15 & $*$ \\
& Mean \pm & $46.87 \quad \pm$ & 54.08 & $63.89 \pm 6$ & \\
& SD. & 3.67 & \pm 5.94 & .11 & \\
\hline
\end{tabular}

**highly statistically significant /*statistically significant.

Table (3): Comparison of area percent and optical density of CD 105 in different histopathological grades.

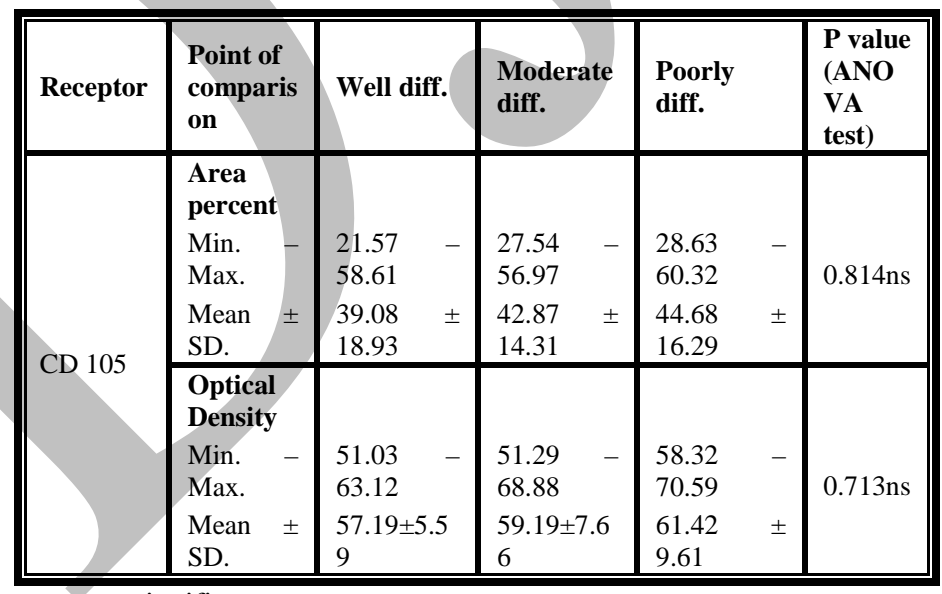

ns=not significant

CD105 expression in Correlation with the Histological Grading of OSCC cases.

The difference in the mean CD105 area percent and mean optical density between the well, moderately and poorly differentiated groups was not statistically significant at $(\mathrm{P}>0.05)$.

\section{DISCUSSION}

In the present study, males (65\%) were more commonly affected than females (35\%). This was in accordance to many previous studies that supported the fact that oral cancer is more common in males than in females. As the habits of alcohol drinking and tobacco smoking is more prevalent in males than in females.(9-11)

Those results were in contrast to those found by Durr et al(12) who stated that the proportion of oral cavity cancer cases in women increased worldwide especially among young white individuals in western countries(12). The alteration of the sex distribution is likely due to the gap is narrowing in habits such as smoking and drinking alcohol became more socially amongst women (13). 
The clinical distribution of OSCC in the current study revealed that the tongue was the most prevalent site of occurrence for OSCC (30\%) followed by the alveolar mucosa (25\%). These results were in accordance with many investigators who reported that the tongue was the most common site for OSCC $(10,11,14,15)$.These results are consistent with the previous studies which revealed that oral SCC affects the tongue in $20 \%-40 \%$ of cases and the floor of the mouth in $15 \%$ - $20 \%$ of the cases, and together these sites account for about $50 \%$ of all cases of oral SCC(16). The gingivae, palate, retromolar areas and the buccal and labial mucosa are oral sites less frequently affected (17).

The results were in contrast to those found by Iamaroon et al that in some other Asian countries such as Taiwan, India and Thailand have found that buccal mucosa was a common location which has strongly associated with betel quid chewing habit regionally $(18,19)$.

Regarding the histological grading of the tumor, the present research showed that most of the OSCC cases included were of the moderately differentiated type (65\%), followed by the well differentiated type (25\%), while the least common type was the poorly differentiated (10\%). This was in accordance to almost all of the studies which supported the fact that moderately differentiated was the most common type of OSCC. Jerjes et al, (11) Kanke et al, (20) and Chen et al (21) all found that moderately differentiated was the most common type of OSCC.

The expression of the human Ki-67 protein is strictly associated with cell proliferation. It is considered to be a fundamental biological process because of the role it plays in the growth and maintenance of tissue homeostasis. It is well understood that transition of the normal oral epithelium to dysplasia and to malignancy is featured by increased cell proliferation. The Ki-67protein as a molecular marker of proliferating cells has been extensively examined in oral epithelial dysplasia and oral squamous cell carcinoma (OSCC), and the number of proliferating cells increased according to the grade of malignancy $(22,23)$.

In this study, Ki-67 protein was expressed in all cases of OSCC. This goes with the results of Olimid et al (24) who stated that the Ki-67 immunoexpression analysis indicated positivity in all cases, but with different percentage index (PI), which correlated with the degree of differentiation of neoplasia.

The results of the present study showed that, the immunoreaction of $\mathrm{Ki}-67$ was seen in the nucleus of the malignant epithelial cells. This is in accordance with the results of J. Carlos de Vicente et al and Costa et al, $(4,25)$. Furthermore Gerdes et al (26) stated, that the antigen was present on chromosomes in all phases of mitosis and within the nucleus of proliferating cells in interphase. Since Ki-67 did not react with cells in a resting stage, as the Ki-67reactive nuclear antigen is not expressed during the Go phase of the cell cycle. Thus, Ki-67 might become an important tool for reliable and rapid determination of proliferation rates of malignant tumors.

In this research, the immunoreaction of Ki-67 was seen in both cytoplasm and nucleus (total cell reactivity) of the malignant epithelial cells.

In the current study, the positive immunoreactivity for Ki-67 was observed in all the control sections. This is consistent with the findings reported in previous studies that showed that Normal oral mucosa showed the lowest expression and controlled proliferation rate but with a continuous proliferative capacity for Ki-67 located mainly in the suprabasal compartment. Also there was a characteristic absence of protein expression in the basal layer for Ki-67 (27). The nuclear antigen detected by Ki-67 was also found to be present in a majority of cells of all the human cell lines investigated.

Gerdes et al, (26) stated that In addition to nuclear staining, the basal cells showed weak, but distinct labelling of the cytoplasm; both nuclear and cytoplasmic staining was also observed in epidermis.

In present study, the difference of Ki-67 staining was statistically significant in different histological grades of OSCC. The well differentiated type was the lowest in Mean optical density \% (MOD\%) and Mean Area \%(MA\%), while the poorly differentiated type was the highest in MOD\% and MA\%.

This is in accordance with the results of Olimid et al (24) on OSCC who found that the Ki-67 immunoreaction in well-differentiated OSCC revealed a medium Ki-67 of 22\%, in moderately differentiated OSCC Ki-67 was 32\%, and a medium value of $53 \%$ in case of poorly differentiated carcinomas.

In the current investigation, the MA\% and MOD\% of Ki67 immunoexpression was higher in the poorly differentiated type followed by the moderate, and finally the well differentiated type. The difference between these grades reached significant value.

Dwivedi, et al (28) found that in well differentiated OSCC, the expression was observed in peripheral areas of islands than the central areas of squamous maturation. This suggested that less differentiated cells are located in the peripheral layer and the central cells are highly differentiated with the ability of keratinization, thus, no expression of Ki-67 was observed in the central cells of the tumor island.

In moderately differentiated OSCC, Ki-67expression when observed in both tumor islands and diffuse staining in other areas. The overall staining of Ki-67 in moderately differentiated tumors was more increased quantitatively than well differentiated whereas in poorly differentiated OSCC the staining of Ki-67 was diffuse and more intense as the cells were less differentiated and in more proliferating phase. The expression of Ki-67 is correlated with the grading of OSCC as it describes the growth fraction of the tumor (29).

CD105, also known as endoglin 8, is a good marker for the measurement of microvascular density (MVD). It is an accessory receptor for transforming growth factor beta (TGF- $\beta$ ) and its expression is up-regulated in actively proliferating endothelial cells. Endoglin has been suggested as an appropriate marker for tumor-related angiogenesis and neovascularization (30). Quantification of tissue angiogenesis can be made by counting microvasculature in a given area by immunohistochemical staining (31).

In the current study, the positive immunoreactivity for CD105 was observed in all the control sections. This is consistent with the findings reported in previous studies. (6, 32-35). Similarly Mărgăritescu et al (25) found that in normal oral mucosa and in the resected tumor edges microvessels rarely expressed CD105 and staining was faint and weak. These vessels were mainly located immediately underneath the epithelium.

In this study, CD105 was expressed in all cases of OSCC. This is in agreement with previous studies which 
stated that (36) endoglin is a powerful marker of neovascularization in solid malignancies. The results of the present study showed that staining is very selective for the blood vessel endothelium. Endoglin reacts specifically with angiogenic endothelial cells. This is in accordance with the results of Mărgăritescu et al(25) who concluded in his studies that endoglin stained intensively intratumoral and invading tumoral front vessels with high sensitivity, whereas vessels in non-neoplastic tissue were not or were weakly expressing CD105.

In the present research, The OSCC cases showed intense and abundant expression of CD105. MA\% and MOD\% were calculated. MA \% reach significant value, while the MOD\% appeared not statistically significant.

In the present study, it was found that there is no significant correlation between different grades of oral squamous cell carcinoma in expression of CD105.These results were in accordance to the results of Mărgăritescu et al (25) which stated that CD105 statistical analysis did not show any significant association of MVD with age, sex, primary tumor's location, clinical stage or differentiation grade. On the other hand, they were not supported by Tadbir et al(22) who found that there was a decreasing level of CD105 at higher grades of differentiation. In both the intratumoral and invasive front regions, as the clinical stage of the disease progressed, the levels of CD105 expression increased. Likewise, Chien et al,(23) Miyahara et al(37), reported the same results. However Mărgăritescu et al.(25) did not observe any association.

\section{CONCLUSION}

Ki-67 67 and CD105 can be detected immunohistochemically in OSCC biopsies. The intensity of their expression is directly proportional to the histopathological grading of the disease. Additionally, the fraction of Ki-67positive tumor cells (the Ki-67 labeling index) is often correlated with the clinical course of OSCC. CD105 microvessel density can be a useful factor for predicting the course of oral squamous cell carcinoma

\section{CONFLICT OF INTEREST}

The authors declare that they have no conflicts of interest.

\section{REFERENCES}

1. Katakura A, Yamamoto N, Sakuma T, Sugahara K, Onda T, Noguchi S, et al. A screening test for oral cancer using saliva samples: Proteomic analysis of biomarkers in whole saliva. J Oral Maxillofac Surg Med Pathol. 2013;27:1-5.

2. Siegel RL, Miller KD, Jemal A. Cancer statistics, 2016. CA Cancer J Clin. 2016;66:7-30.

3. Kervancioglu E, Kosan M, Erinanc H, Gonulalan U, Oguzulgen AI, Coskun EZ, et al. Predictive values of vascular endothelial growth factor and microvessel-density levels in initial biopsy for prostate cancer. Kaohsiung J Med Sci. 2016;32:74-9.

4. Kulasinghe A, Perry C, Warkiani ME, Blick T, Davies A, O'Byrne K, et al. Short term ex-vivo expansion of circulating head and neck tumour cells. Oncotarget. 2016;7:60101-9.

5. de Moraes M MMC, de Almeida Freitas R, Galvão HC. Cell proliferation markers in oral squamous cell carcinoma. J Mol Biomark Diagn. 2012;S2.
6. Zhu Y, Yang QC, Liu HB, Liu MH, Shen Y, Zhang XJ. [Expression and significance of hypoxia-inducible factor 1alpha protein and microvessel density marked by CD105 in cervical carcinoma]. Zhonghua Bing Li Xue Za Zhi. 2008;37:264-5.

7. Tumuluri V, Thomas GA, Fraser IS. Analysis of the Ki-67 antigen at the invasive tumour front of human oral squamous cell carcinoma. J Oral Pathol Med. 2002;31:598604.

8. Kurokawa H, Zhang M, Matsumoto S, Yamashita Y, Tanaka $\mathrm{T}$, Tomoyose $\mathrm{T}$, et al. The relationship of the histologic grade at the deep invasive front and the expression of Ki-67 antigen and p53 protein in oral squamous cell carcinoma. J Oral Pathol Med. 2005;34:6027.

9. Acharya S, Sivakumar AT, Shetty S. Cervical lymph node metastasis in oral squamous cell carcinoma: a correlative study between histopathological malignancy grading and lymph node metastasis. Indian J Dent Res. 2013;24:599604.

10. Girardi FM, Zanella VG, Kroef RG. Correlation between clinical and pathological data and surgical margins in patients with squamous cell carcinoma of the oral cavity. Braz J Otorhinolaryngol. 2013;79:190-5.

11. Jerjes W, Upile T, Petrie A, Riskalla A, Hamdoon Z, Vourvachis $\mathrm{M}$, et al. Clinicopathological parameters, recurrence, locoregional and distant metastasis in 115 T1T2 oral squamous cell carcinoma patients. Head Neck Oncol. 2010;2:9.

12. Durr ML, van Zante A, Li D, Kezirian EJ, Wang SJ. Oral tongue squamous cell carcinoma in never-smokers: analysis of clinicopathologic characteristics and survival. Otolaryngol Head Neck Surg. 2013;149:89-96.

13. Li R, Koch WM, Fakhry C, Gourin CG. Distinct epidemiologic characteristics of oral tongue cancer patients. Otolaryngol Head Neck Surg. 2013;148:792-6.

14. Pires FR, Ramos AB, Oliveira JB, Tavares AS, Luz PS, Santos TC. Oral squamous cell carcinoma: clinicopathological features from 346 cases from a single oral pathology service during an 8-year period. J Appl Oral Sci. 2013;21:460-7.

15. Warnakulasuriya S. Global epidemiology of oral and oropharyngeal cancer. Oral Oncology. 2009;45:309-16.

16. Bello IO, Soini Y, Salo T. Prognostic evaluation of oral tongue cancer: means, markers and perspectives (I). Oral Oncol. 2010;46:630-5.

17. Warnakulasuriya S. Global epidemiology of oral and oropharyngeal cancer. Oral Oncol. 2009;45:309-16.

18. Chang TS, Chang CM, Ho HC, Su YC, Chen LF, Chou P, et al. Impact of young age on the prognosis for oral cancer: a population-based study in Taiwan. PLoS One. 2013;8:e75855.

19. Krishna A, Singh RK, Singh S, Verma P, Pal US, Tiwari S. Demographic risk factors, affected anatomical sites and clinicopathological profile for oral squamous cell carcinoma in a north Indian population. Asian Pac J Cancer Prev. 2014;15:6755-60.

20. Kanke M, Fujii M, Kameyama K, Kanzaki J, Tokumaru Y, Imanishi Y, et al. Role of CD44 variant exon 6 in invasion of head and neck squamous cell carcinoma. Arch Otolaryngol Head Neck Surg. 2000;126:1217-23.

21. Chen YK, Huang HC, Lin LM, Lin CC. Primary oral squamous cell carcinoma: an analysis of 703 cases in southern Taiwan. Oral Oncol. 1999;35:173-9. 
22. Tadbir AA, Ashraf MJ, Moradi M. Clinicopathological Significance of CD105 Expression in Squamous Cell Carcinoma of the Oral Cavity. Middle East J Cancer. 2014;5:7-12.

23. Chien CY, Su CY, Hwang CF, Chuang HC, Hsiao YC, Wu $\mathrm{SL}$, et al. Clinicopathologic significance of CD105 expression in squamous cell carcinoma of the hypopharynx. Head Neck. 2006;28:441-6.

24. Olimid DA, Simionescu CE, Margaritescu C, Florescu A. Immunoexpression of Ki67 and cyclin D1 in oral squamous carcinomas. Rom J Morphol Embryol. 2012;53(3 Suppl):795-8.

25. Margaritescu C, Pirici D, Stinga A, Simionescu C, Raica M, Mogoanta L, et al. VEGF expression and angiogenesis in oral squamous cell carcinoma: an immunohistochemical and morphometric study. Clin Exp Med. 2010;10:209-14.

26. Gerdes J, Schwab U, Lemke H, Stein H. Production of a mouse monoclonal antibody reactive with a human nuclear antigen associated with cell proliferation. Int J Cancer. 1983;31:13-20.

27. Torres-Rendon A, Roy S, Craig GT, Speight PM. Expression of Mcm2, geminin and Ki67 in normal oral mucosa, oral epithelial dysplasias and their corresponding squamous-cell carcinomas. Br J Cancer. 2009;100:1128-34.

28. Dwivedi N, Chandra S, Kashyap B, Raj V, Agarwal A. Suprabasal expression of Ki-67 as a marker for the severity of oral epithelial dysplasia and oral squamous cell carcinoma. Contemp Clin Dent. 2013;4:7-12.

29. Blank S, Deck C, Dreikhausen L, Weichert W, Giese N, Falk C, et al. Angiogenic and growth factors in gastric cancer. J Surg Res. 2015;194:420-9.

30. Nassiri F, Cusimano MD, Scheithauer BW, Rotondo F, Fazio A, Yousef GM, et al. Endoglin (CD105): a review of its role in angiogenesis and tumor diagnosis, progression and therapy. Anticancer Res. 2011;31:2283-90.

31. Himani B, Meera S, Abhimanyu S, Usha R. Ki-67 Immunostaining and its Correlation with Microvessel Density in Patients with Mutiple Myeloma. Asian Pac J Cancer Prev. 2016;17:2559-64.

32. Kerbel RS. Tumor angiogenesis: past, present and the near future. Carcinogenesis. 2000;21:505-15.

33. Chopra A. 89Zr-Labeled anti-CD105 (endoglin) chimeric monoclonal antibody TRC105 linked to IRDye 800CW. Molecular Imaging and Contrast Agent Database (MICAD). Bethesda (MD)2004.

34. Zhang Y, Yang Y, Hong H, Cai W. Multimodality molecular imaging of CD105 (Endoglin) expression. Int J Clin Exp Med. 2011;4:32-42.

35. Aristorena M, Blanco FJ, de Las Casas-Engel M, OjedaFernandez L, Gallardo-Vara E, Corbi A, et al. Expression of endoglin isoforms in the myeloid lineage and their role during aging and macrophage polarization. J Cell Sci. 2014;127:2723-35.

36. Bernabeu C, Lopez-Novoa JM, Quintanilla M. The emerging role of TGF-beta superfamily coreceptors in cancer. Biochim Biophys Acta. 2009;1792:954-73.

37. Miyahara M, Tanuma J, Sugihara K, Semba I. Tumor lymphangiogenesis correlates with lymph node metastasis and clinicopathologic parameters in oral squamous cell carcinoma. Cancer. 2007;110:1287-94. 